\title{
Reappraising the Need for Bulk Heterojunctions in Polymer-Fullerene Photovoltaics: The Role of Carrier Transport in All-Solution-Processed P3HT/PCBM Bilayer Solar Cells
}

\author{
Alexander L. Ayzner, Christopher J. Tassone, Sarah H. Tolbert,* and Benjamin J. Schwartz* \\ Department of Chemistry and Biochemistry and California Nanosystems Institute, University of California, \\ Los Angeles, Los Angeles, California 90095-1569 USA
}

Received: May 30, 2009; Revised Manuscript Received: September 15, 2009

\begin{abstract}
The most efficient organic solar cells produced to date are bulk heterojunction (BHJ) photovoltaic devices based on blends of semiconducting polymers such as poly(3-hexylthiophene-2,5-diyl) (P3HT) with fullerene derivatives such as [6,6]-penyl- $\mathrm{C}_{61}$-butyric-acid-methyl-ester $(\mathrm{PCBM})$. The need for blending the two components is based on the idea that the exciton diffusion length in polymers like P3HT is only $\sim 10 \mathrm{~nm}$, so that the polymer and fullerene components must be mixed on this length scale to efficiently split the excitons into charge carriers. In this paper, we show that the BHJ geometry is not necessary for high efficiency, and that all-solution-processed P3HT/PCBM bilayer solar cells can be nearly as efficient as BHJ solar cells fabricated from the same materials. We demonstrate that $o$-dichlorobenzene (ODCB) and dichloromethane serve nicely as a pair of orthogonal solvents from which sequential layers of P3HT and PCBM, respectively, can be spin-cast. Atomic force microscopy, various optical spectroscopies, and electron microscopy all demonstrate that the act of spin-coating the PCBM overlayer does not affect the morphology of the P3HT underlayer, so that our spin-cast P3HT/PCBM bilayers have a well-defined planar interface. Our fluorescence quenching experiments find that there is still significant exciton splitting in P3HT/PCBM bilayers even when the P3HT layer is quite thick. When we fabricated photovoltaic devices from these bilayers, we obtained photovoltaic power conversion efficiencies in excess of 3.5\%. Part of the reason for this high efficiency is that we were able to separately optimize the roles of each component of the bilayer; for example, we found that thermal annealing has relatively little effect on the nature of P3HT layers spin-cast from ODCB, but that it significantly increases the crystallinity and thus the mobility of electrons through PCBM. Because the carriers in bilayer devices are generated at the planar P3HT/PCBM interface, we also were able to systematically vary the distance the carriers have to travel to be extracted at the electrodes by changing the layer thicknesses without altering the bulk mobility of either component or the nature of the interfaces. We found that devices have the best fill-factors when the transit times of electrons and holes through the two layers are roughly balanced. In particular, we found that the most efficient devices are made with P3HT layers that are about four times thicker than the PCBM layers, demonstrating that it is the conduction and the extraction of electrons through the fullerene that ultimately limit the performance of both bilayer and BHJ devices based on the P3HT/ PCBM material combination. Overall, we believe that polymer-fullerene bilayers provide several advantages over BHJ devices, including reduced carrier recombination and a much better degree of control over the properties of the individual components and interfaces during device fabrication.
\end{abstract}

\section{Introduction}

Thin film photovoltaics (PVs) based on blends of conjugated polymers as electron donors and fullerenes as electron acceptors have been the subject of intense research owing to the ease with which they can be fabricated into inexpensive plastic solar cells. ${ }^{1-3}$ When blended together, conjugated polymers and fullerenes phase segregate on nanometer length scales, producing a bicontinuous interpenetrating network of the polymer and fullerene components, which is often referred to as a bulk heterojunction (BHJ). ${ }^{4,5}$ Light incident on BHJ solar cells is primarily absorbed by the $\pi$-conjugated polymer, leading to the creation of strongly bound excitons. Literature reports have estimated that excitons can diffuse only over distances of $\sim 10$ $n m .{ }^{67}$ If the polymer and fullerene components are phasesegregated on this same length scale, then essentially every

* Corresponding authors. (B. J. S.) E-mail: schwartz@chem.ucla.edu. Tel.: (310) 206-4113. Fax: (310) 206-4038. (S. H. T.) E-mail: tolbert@ chem.ucla.edu. Tel.: (310) 206-4767. Fax: (310) 206-4038. exciton can diffuse to within charge-transfer range of a fullerene molecule during its lifetime, resulting in exciton splitting and formation of polaron pairs ${ }^{8-10}$ with near-unit quantum yield. ${ }^{11-13}$ These coulombically-bound charge pairs are then separated because of a combination of electric potential and concentration gradients ${ }^{14}$ and eventually collected at the electrodes to produce a photocurrent in the external circuit. To date, BHJ solar cells based on the combination of the regioregular polymer poly(3hexylthiophene-2,5-diyl) (P3HT) and the fullerene derivative [6,6]-penyl- $\mathrm{C}_{61}$-butyric-acid-methyl-ester (PCBM) have reached power conversion efficiencies exceeding $\sim 5 \%,{ }^{15-17}$ with even higher efficiencies obtained for devices based on redderabsorbing conjugated polymers and/or fullerene derivatives. ${ }^{18}$

Even though polymer-based BHJ solar cells have achieved quite respectable power conversion efficiencies, questions still remain regarding the fundamental processes that ultimately limit device performance. For example, there is still significant argument as to whether the mobility of holes in the polymer component or electrons in the fullerene component of the BHJ 
cell is what limits device performance. ${ }^{19-23}$ It is well known that thermal annealing improves the power conversion efficiency of polymer-based BHJ photovoltaics, but the effects of annealing on carrier mobility in the individual BHJ components and the way annealing affects the degree of phase segregation also have been the subject of debate. ${ }^{19,20}$ Finally, because BHJs have a complex, difficult-to-characterize nanoscale morphology, ${ }^{15,24-28}$ there have been no systematic studies investigating how the transit times for electrons and holes on the two components affect the general shape of the device current-voltage response under solar illumination.

In this paper, we address these fundamental issues in polymer-fullerene photovoltaic systems by removing the complexity associated with the nanoscale architecture of BHJs and focusing on fully solution-cast planar P3HT/PCBM bilayer solar cells. Although the bilayer geometry has not been popular for polymer-based solar cells on the basis of the argument that the smaller interfacial area between the donor and the acceptor in bilayers results in reduced exciton splitting relative to that in BHJ's, we find that we still get significant exciton harvesting even when the polymer component of the bilayer is optically thick. The facts that exciton splitting in bilayers is still efficient and that segregating the donor and acceptor layers drastically reduces bimolecular recombination ${ }^{29}$ has allowed us to produce all-solution-processed bilayer PV cells with fill-factors reaching $70 \%$ and power conversion efficiencies in excess of 3.5\%. Perhaps more importantly, by separating the layers, we have been able to elucidate much of the physics that underlies the operation of polymer-fullerene solar cells. We find that the increase in crystallinity of the PCBM component is largely responsible for the improvement in power conversion efficiency that occurs upon thermal annealing. In addition, we have been able to directly investigate how the difference between the electron and hole transit times affects the shape of the device current-voltage curve. We find not only that it is critical to balance the electron and hole transit times to produce devices with optimal efficiency, but also that electron transport in the fullerene component is what limits the performance of both P3HT/PCBM bilayer and BHJ solar cells.

Beyond understanding the role of the separate components and interfaces in polymer-fullerene photovoltaics, we also show in this paper that there are additional advantages to being able to form bilayer solar-cells cast entirely from solution. First, we demonstrate that there is a set of so-called orthogonal solvents that allows sequential spin-coating of polymer and fullerene layers to produce bilayers: when the right solvent is chosen for spin-casting the fullerene overlayer, there are no changes in the surface morphology of the polymer underlayer, so that there is a sharp, well-defined interface between the two layers. This allows the production of bilayer solar cells with an ease of fabrication that rivals that of BHJ devices and significantly surpasses that of devices in which one of the components must be thermally evaporated under high vacuum. The ability to create solution-processed bilayers also enables the use of organic electron acceptors that may not survive thermal evaporation. Second, because the two components of bilayer films are deposited separately, the absorption spectrum and nanometerscale morphology of both the polymer and the fullerene layers can be controlled and optimized independently by using techniques such as thermal or solvent annealing. Finally, depositing the fullerene top layer from solution offers the possibility to achieve efficient exciton dissociation without adversely affecting the mobilities of the carriers being transported in either the polymer or fullerene layers. Thus, we believe that these advantages make the bilayer geometry a serious contender for the future production of large-scale, efficient polymer-fullerene-based solar cells.

\section{Experimental Section}

For the production of bilayer photovoltaic devices, there is an inherent difficulty associated with spin-coating sequential layers because most conjugated organic molecules are soluble in similar solvents, so that spin-coating a film on top of an organic underlayer usually results in significant redissolution of the bottom layer. Thus, bilayer devices are often produced with one or both layers deposited by thermal evaporation, which limits the device area and restricts the choice of active organic molecules to those that do not decompose during sublimation. One recent alternate approach demonstrated the production of P3HT/PCBM bilayer solar cells by transferring a PCBM layer onto precoated P3HT substrates by using a poly(dimethylsiloxane) (PDMS) stamp, resulting in power conversion efficiencies of $\sim 1.5 \%{ }^{30}$ Another recent alternate approach involved photocross-linking a derivative of $\mathrm{P} 3 \mathrm{HT}$ to render it insoluble, so that a PCBM overlayer could be spun on top, producing devices with power conversion efficiencies of $\sim 2 \% .{ }^{31}$ In contrast, fabrication of fully solution-processed bilayer cells via spincoating of both components requires finding a set of so-called orthogonal solvents ${ }^{32}$ such that the solvent used to spin-coat the fullerene overlayers does not affect the morphology of the polymer underlayers. For P3HT/PCBM devices, we find that the common organic solvent dichloromethane (DCM) meets this requirement: PCBM is sufficiently soluble in DCM that it is possible to spin-coat PCBM layers on top of P3HT, and as we show below, P3HT is so sparingly soluble in DCM that there is negligible redissolution of the P3HT underlayer during spincoating of the PCBM overlayer.

We prepared our P3HT/PCBM bilayer solar cells by starting with prepatterned indium-doped tin oxide (ITO; TFD sales) substrates that were first cleaned by successive sonications in detergent solution, deionized water, acetone, and finally isopropanol for approximately $10 \mathrm{~min}$ each. The substrates were then blown dry with Ar and briefly treated with an air plasma (200 mTorr, $10 \mathrm{~min}$ ) prior to spin-coating a thin $(\leq 50$ $\mathrm{nm})$ poly(ethylenedioxythiophene):poly(styrenesulfonic acid) (PEDOT:PSS, Baytron P VP A1 4083) layer at $5000 \mathrm{rpm}$ for $60 \mathrm{~s}$. The PEDOT:PSS-coated slides were then baked on a digitally controlled hot plate in a nitrogen atmosphere for 20 min at $140{ }^{\circ} \mathrm{C}$. We prepared solutions of regioregular P3HT (Rieke Metals, 90-93\% regioregular) in o-dichlorobenzene (ODCB) at concentrations of 10, 15, 20, 22.5, 25, and $27.5 \mathrm{mg} /$ $\mathrm{mL}$. These solutions were heated to $55^{\circ} \mathrm{C}$ for several hours in a nitrogen atmosphere before being cooled to room temperature and spin-cast onto the PEDOT:PSS-coated substrates at 1000 rpm for $90 \mathrm{~s}$, producing P3HT films with thicknesses of $50 \pm$ 2, $80 \pm 2$, and $115 \pm 2 \mathrm{~nm}$, from the 10,15 , and $20 \mathrm{mg} / \mathrm{mL}$ solutions, respectively, as measured by using a profilometer (Dektak). Thicknesses for films spun from higher-concentration solutions were estimated using a thickness vs. optical density calibration curve. Freshly-spun P3HT films were allowed to dry in a $\mathrm{N}_{2}$ atmosphere for at least $20 \mathrm{~min}$ prior to spin-coating the PCBM overlayer. We then prepared solutions of PCBM (NanoC) in DCM at concentrations of 5 and $10 \mathrm{mg} / \mathrm{mL}$; the $10 \mathrm{mg} /$ $\mathrm{mL}$ solution was briefly heated at $40{ }^{\circ} \mathrm{C}$ to ensure maximal dissolution. We found the solubility limit of PCBM in DCM to be at or just under $10 \mathrm{mg} / \mathrm{mL}$; thus, only the $10 \mathrm{mg} / \mathrm{mL}$ solution was filtered prior to spin-coating. We then spin-cast the PCBM solutions at $4000 \mathrm{rpm}$ for $10 \mathrm{~s}$ onto the P3HT films from the 
previous step, producing PCBM film thicknesses of $22 \pm 2$ and $34 \pm 2 \mathrm{~nm}$, respectively. At a rate of less than $5 \AA / \mathrm{s}$, we then evaporated a cathode consisting of $20 \mathrm{~nm}$ of Ca followed by a $20 \mathrm{~nm} \mathrm{Al}$ protective overlayer onto the completed bilayers through a shadow mask, resulting in active device areas of 6.5 $\mathrm{mm}^{2}$.

We measured the photovoltaic performance of our devices in an argon atmosphere by using a Keithley 2400 source meter. A xenon arc lamp equipped with a liquid light guide (Oriel) and an AM-1.5 filter was used as the excitation source; the intensity of the incident light on the devices was adjusted to $100 \mathrm{~mW} / \mathrm{cm}^{2}$, as determined by using a calibrated silicon photodiode. We calculated a spectral mismatch factor ${ }^{33}$ for our setup of nearly unity. To investigate the effects of thermal annealing on device performance, we placed bilayer devices prior to cathode deposition on a digitally controlled hot plate at $150{ }^{\circ} \mathrm{C}$ for $20 \mathrm{~min}$ in an $\mathrm{Ar}$ atmosphere; the films were covered with a shallow Petri dish during annealing to help ensure uniform heating. At the end of the $20 \mathrm{~min}$ annealing cycle, the films were rapidly cooled by placing them onto a room-temperature metal surface.

We collected photoluminescence (PL) spectra from our bilayer films in air at $22.5^{\circ}$ with respect to the excitation beam with the sample positioned at $70^{\circ}$ with respect to the excitation axis. We kept the slit widths and integration times constant for all of our experiments and also normalized all of the PL spectra displayed by the optical density of the sample at the $530 \mathrm{~nm}$ excitation wavelength and corrected for the detector and monochromator responses so that the relative intensities of the different PL spectra presented below are meaningful.

Atomic force microscopy (AFM) was carried out by using a Nanoscope V Dimension 5000 (Veeco Digital Instruments) in ambient conditions. Antimony $n$-doped silicon cantilevers (TESPW, Veeco Probes) with spring constants of $42 \mathrm{~N} / \mathrm{m}$, first longitudinal resonance frequencies between $230-410 \mathrm{kHz}$, and nominal tip radii of $8 \mathrm{~nm}$ were employed in tapping mode. Simultaneous height and phase images were acquired and reproduced across multiple samples. To image the P3HT polymer layer after bilayer fabrication, the PCBM overlayer was removed by soaking the bilayer films in cyclohexane $(\mathrm{CH})$ for several days in the dark under ambient conditions and then drying the films under vacuum before performing the measurements; as shown below, we found no spectroscopic or AFM evidence for any remaining fullerene following such treatment.

To characterize the PCBM overlayers in our bilayers by X-ray diffraction (XRD), we carried out two-dimensional (2-D) grazing incidence XRD at the Stanford Synchrotron Radiation Light Source on beamline 11-3 with a wavelength of $0.9742 \AA$. Data were collected on both pure PCBM films spun from DCM onto single-crystal Si substrates and on P3HT/PCBM bilayers. Both samples gave similar diffraction data: diffuse low-intensity diffraction for unannealed films and a series of somewhat sharper peaks in films that had been thermally annealed. Because of the very strong P3HT diffraction in the bilayers, however, the PCBM diffraction in the bilayer films was harder to see; thus, we only show the data collected for pure PCBM films below. Because the 2-D PCBM diffraction images did not show any preferred orientation, we radially integrated the data to produce the one-dimensional patterns shown below, making it easier to clearly visualize the degree of crystallinity in each film.

\section{Results and Discussion}

Although layers of PCBM have been spin-cast from DCM onto P3HT films in the past, ${ }^{34,35}$ there has been essentially no work investigating either the quality of the PCBM films produced by spin-coating or the effects of spinning the PCBM top layer onto the P3HT underlayer. Thus, we begin this section with a detailed examination of the morphology of our spin-cast P3HT/PCBM bilayers. We show that the act of spinning pure DCM solvent has a negligible effect on the P3HT underlayer, and that PCBM layers can be deposited and removed without significantly altering the surface topography of the underlying P3HT film. We also show that the initially deposited PCBM film is largely amorphous, but that the PCBM layer becomes partially nanocrystalline upon thermal annealing. We then turn to study the steady-state photophysics of our P3HT-PCBM bilayers, where PL spectroscopy allows us to investigate the nature of how well PCBM overlayers quench excitons in the P3HT underlayers. We then conclude this section with a detailed investigation of the performance of solar cells based on P3HT/ PCBM bilayers.

A. Physical Characterization of P3HT/PCBM SolutionProcessed Bilayers. One of the real advantages to solutionprocessed bilayers is that, as long as the two layers are distinct and do not significantly intermix, the morphology and other properties of each layer can be studied independently as the processing conditions are varied. In this subsection, we show, by using a combination of AFM and optical measurements, that the P3HT/PCBM solution-processed bilayers we make have a sharp ( $\sim 1-\mathrm{nm}$ roughness) interface between the P3HT and PCBM components, a conclusion that is also supported by electron microscopy images on cross sections of our bilayers that are presented in the Supporting Information. ${ }^{36}$ We then use a combination of AFM and XRD to investigate the effects of thermal annealing on the individual P3HT and PCBM components of the bilayers and show that the primary effect of annealing is to increase the crystallinity of the PCBM overlayer.

1. Sharp Interface of P3HT/PCBM Solution-Processed Bilayers. Because we are preparing our bilayers by spin-casting the PCBM overlayer from DCM, it is important to ensure that the P3HT underlayer is not dissolved or altered by the DCM solvent used to spin the overlayer. To do this, we started by simply placing a significant amount of P3HT powder into DCM solvent. After stirring for several days, the vast majority of the polymer remained undissolved. The fact that the UV-visible absorption of the solution was significantly blue-shifted from that of P3HT solutions in good solvents such as ODCB indicates that only a small amount of low-molecular-weight and/or regiorandom material had dissolved in the DCM. After several washes, we found that DCM solvent left in contact with P3HT powder was only very faintly colored. Thus, we can conclude that, with the possible exception of regiorandom impurities or some very-low-molecular-weight material, regioregular P3HT is essentially insoluble in DCM. In the spectroscopic data shown below, we prepared the film samples by using the DCM-washed P3HT powder; however, we found that the performance of our bilayer solar cells did not depend on whether the P3HT powder was washed in DCM.

To verify that the use of DCM solvent for deposition of the PCBM overlayer does not affect the morphology of the P3HT underlayer, we present tapping-mode AFM phase images of the initially deposited P3HT layer as it undergoes the several steps of processing needed to fabricate a bilayer in Figure 1. An image of the surface of a film of pure P3HT cast from ODCB is shown in Figure 1A. The film's surface is composed of rice-like nanoscale crystallites with an average diameter of $13.8 \pm 2.4$ $\mathrm{nm}$. To test the effects of spinning an overlayer from a different solvent onto the P3HT film, we spin-cast a drop of pure DCM 

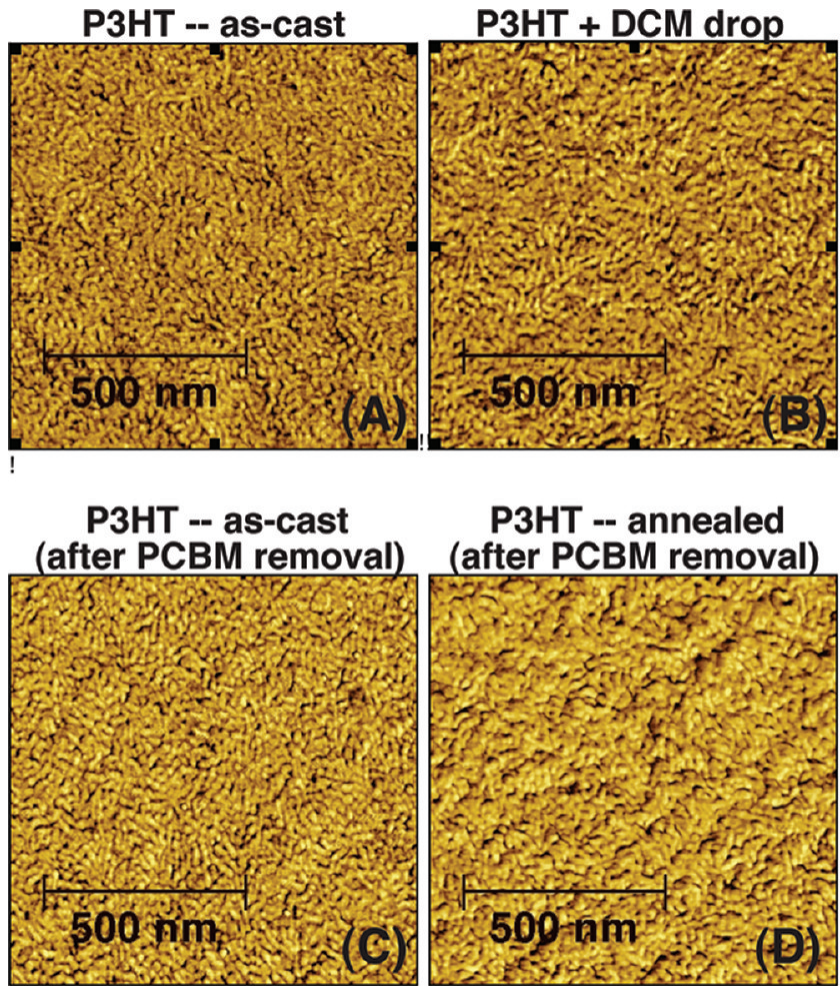

Figure 1. AFM tapping-mode phase images $(1 \mu \mathrm{m} \times 1 \mu \mathrm{m})$ of $(\mathrm{A})$ an-cast P3HT film. The nanocrystalline domains have an average diameter of $\sim 14 \mathrm{~nm}$, and the rms surface roughness is $1.55 \mathrm{~nm}$. (B) an as-cast P3HT film onto which a drop of DCM solvent has been spun. The size of the nanocrystalline domains and surface roughness are identical within the error to the as-cast film shown in panel A. (C) an as-cast P3HT film onto which a PCBM layer had been spun from DCM and then subsequently removed by soaking the bilayer in $\mathrm{CH}$. The size of the P3HT nanocrystalline domains is identical within the noise to the films shown in panels A and B. (D) a P3HT film onto which a PCBM layer had been spun from DCM with the bilayer annealed at $150{ }^{\circ} \mathrm{C}$ for $20 \mathrm{~min}$ following removal of the PCBM overlayer by soaking in $\mathrm{CH}$. The annealing process increases the average size of the P3HT nanocrystallites to $\sim 17 \mathrm{~nm}$, and the rms surface roughness to $2.67 \mathrm{~nm}$. The scale bar in each panel is $500 \mathrm{~nm}$.

onto the P3HT film and remeasured the AFM tapping-mode phase image, shown in Figure 1B. The data make it clear that the morphology of the P3HT underlayer is maintained in the presence of DCM; the average P3HT crystallite diameter remains essentially unchanged at $13.2 \pm 2.4 \mathrm{~nm}$. Spinning DCM solvent onto the P3HT layer also has a negligible effect on the surface roughness of the P3HT film: the root mean square (rms) surface roughness changes from 1.55 to $1.47 \mathrm{~nm}$ upon addition of the spin-cast DCM drop, a difference that is within the batchto-batch variations we observed over multiple measurements. Thus, we can conclude that the addition of DCM does not cause any detectable differences in the surface morphology of P3HT films.

To further verify that creating a bilayer by spin-casting a solution of PCBM in DCM on top of P3HT does not alter the morphology of the underlying P3HT film, we fabricated a P3HT/ PCBM bilayer and then removed the PCBM overlayer by soaking the bilayer in $\mathrm{CH}$ for several days; a tapping-mode AFM phase image of the P3HT layer that remained following removal of the PCBM overlayer is shown in Figure 1C. Like with spincasting a pure DCM drop, spin-casting a PCBM overlayer and then removing it has little effect on the underlying P3HT surface morphology: the diameter of the crystalline grains are $14.00 \pm$ $1.8 \mathrm{~nm}$, which is unchanged within the error of the measurement.

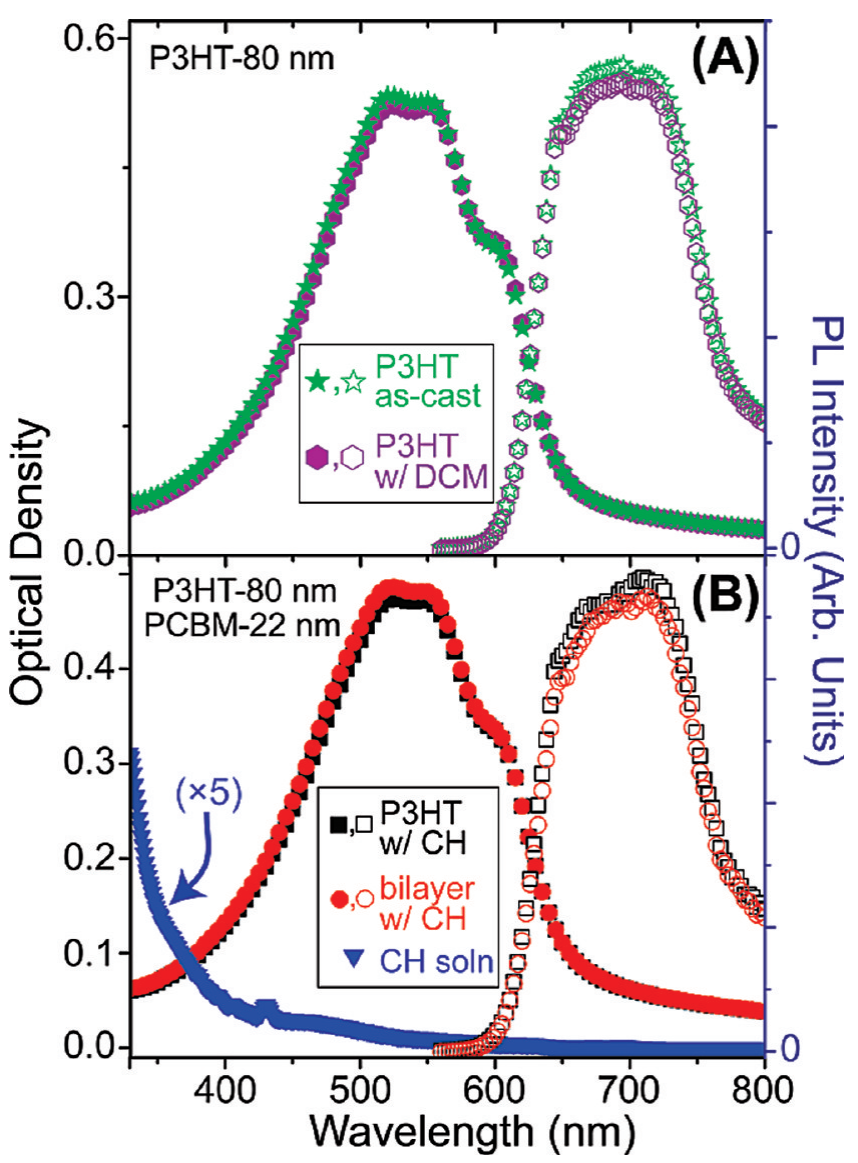

Figure 2. Steady-state absorption spectra (solid symbols, left axes) and PL spectra (open symbols, right axes) of P3HT films at various processing stages in the formation of P3HT/PCBM bilayers. (A) Steadystate spectra of an $80 \mathrm{~nm}$ thick P3HT film as-cast from ODCB (green stars) and the same film onto which a drop of DCM solvent had been spun (purple hexagons). The P3HT powder was pre-washed with DCM to ensure complete removal of a small amount of oligomeric and regiorandom segments. (B) Steady-state spectra of an $80 \mathrm{~nm}$ thick P3HT film spin-cast from ODCB that had been soaked in $\mathrm{CH}$ (black squares) and a $80 \mathrm{~nm}$ P3HT/22 nm PCBM bilayer fabricated as described in the text that had also been soaked in $\mathrm{CH}$ for the same amount of time (red circles). The solid blue squares show the absorption spectrum of the $\mathrm{CH}$ solution that had been used to soak the bilayer; the absorption spectrum of this solution exactly matches that of solution-phase PCBM.

The surface roughness of the P3HT film, on the other hand, does increase slightly to $2.47 \mathrm{~nm}$ upon addition and removal of the PCBM overlayer. A comparison to the surface topography of an as-cast P3HT film that had been soaked in $\mathrm{CH}$ (without first spin-casting a PCBM overlayer), however, shows a nearly identical topography. These results suggest that the small increase in surface roughening seen via AFM comes from soaking the bilayer in $\mathrm{CH}$ and that the act of spin-coating the PCBM overlayer from DCM negligibly changes the underlying P3HT film morphology. This conclusion is also supported by the electron-microscopy results presented in the Supporting Information, ${ }^{36}$ which show that the thickness and surface roughness of the P3HT layer do not change after overcoating with PCBM to produce a bilayer.

Additional evidence that spin-coating a PCBM overlayer from a DCM solution does not significantly alter the structure of the P3HT film underneath is presented in Figure 2, which shows the absorption and PL spectra of pure P3HT films before and after spinning a DCM drop on top of the film (panel A) and the spectra of P3HT and P3HT/PCBM bilayer samples after soaking 
P3HT:PCBM BHJ (as-cast)

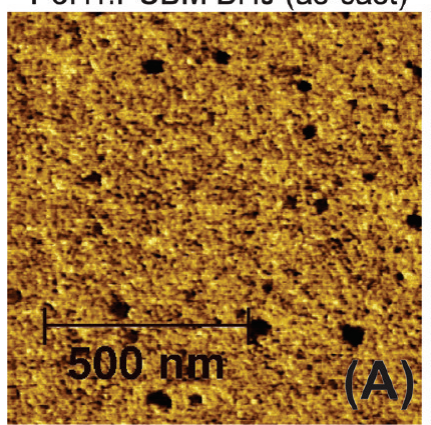

surface roughness $=1.5 \mathrm{~nm}$

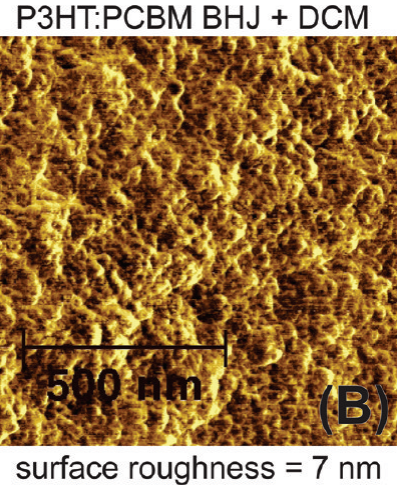

Figure 3. AFM tapping-mode phase images $(1 \mu \mathrm{m} \times 1 \mu \mathrm{m})$ of $(\mathrm{A})$ an $80 \mathrm{~nm}$ thick 1:1 w/w P3HT:PCBM BHJ blend film spin-cast from ODCB. The nanocrystalline P3HT domains seen in Figure 1 are suppressed by the presence of PCBM at the top surface of the film, which breaks up the order of the P3HT chains. The large black features, which correspond to bumps in topography, result from PCBM-rich domains. (B) The same BHJ film shown in panel A onto which a drop of pure DCM solvent had been spun. The new features and $\sim 5$ fold increase in surface roughness result from the removal of PCBM from the blend film by the DCM solvent, leaving behind an open P3HT matrix. These results show clearly that if the PCBM in bilayer structures had interdiffused into the P3HT underlayer, it would have resulted in obvious signatures via AFM.

in $\mathrm{CH}$ (panel B). Figure 2A shows that neither the absorption nor the PL of a P3HT film is affected by spin-coating a drop of DCM solvent on top of the P3HT film, a result consistent with the AFM and electron microscopy ${ }^{36}$ data discussed above. Figure 2B shows that after soaking both a pure P3HT film and a P3HT/ PCBM bilayer in $\mathrm{CH}$ for several days, the PL intensity of the former bilayer film is identical within error to that of the pure P3HT film, indicating that our soaking procedure has effectively removed all of the PCBM from the bilayer. We also see that the solvent used to soak the bilayer exhibits the solution-phase spectrum of PCBM. The fact that both the optical and topographic properties of P3HT films are virtually unaffected by spin-coating the PCBM overlayer provides consistent evidence that the $\mathrm{P} 3 \mathrm{HT} / \mathrm{PCBM}$ interface in our bilayer samples is relatively sharp.

Finally, Figure 3 presents the results of experiments that verify that if interdiffusion of PCBM into the P3HT underlayer had occurred during formation of the bilayer, we would have seen clear signatures of this via AFM. Figure 3A shows a tappingmode AFM phase image of an $80 \mathrm{~nm}$ thick 1:1 w/w P3HT: PCBM BHJ film spin-cast from ODCB. The rice-grain-like structure that is seen at the surface of films of pure P3HT (cf. Figure 1) is suppressed because the presence of PCBM in the film breaks up the nanoscale crystallinity of the P3HT. The dark features in this phase image, which correspond to bumps in topography, indicate that PCBM-rich domains are present at the top surface of the $\mathrm{BHJ}$ film. ${ }^{37}$ The fact that the rice-grainlike structure at the surface of the P3HT underlayers in our bilayers is still clearly visible following deposition and removal of the PCBM overlayer (Figure 1C) is thus additional evidence that PCBM did not diffuse into the P3HT underlayer. Moreover, Figure 3B shows an AFM phase image of an P3HT:PCBM BHJ film that had had a drop of pure DCM solvent spun on top of it; other than the DCM drop, this BHJ film was prepared identically to the one shown in Figure 3A. Because PCBM is highly soluble in DCM, the act of spin-coating DCM onto the $\mathrm{BHJ}$ blend film removes a significant fraction of the PCBM, as verified by absorption and PL spectroscopy similar to that shown above in Figure 2. The removal of PCBM leaves large craters and valleys in the surrounding P3HT matrix that are clearly visible in the AFM image and result in a $\sim 5$ fold increase in the surface roughness of the film. Thus, the data in Figure 3 verify that there would have been obvious topographic signatures if PCBM had significantly interdiffused into the P3HT underlayer during the bilayer fabrication process; therefore, we can be confident that the P3HT/PCBM bilayers we produce have a relatively sharp interface between the two components.

2. Effects of Thermal Annealing on the Morphology of P3HT/PCBM Bilayers. Now that we have established that the $\mathrm{P} 3 \mathrm{HT} / \mathrm{PCBM}$ interfaces in our solution-processed bilayer samples are fairly sharp, we can use AFM to examine the changes in P3HT surface morphology induced by thermal annealing. If we spin-cast a P3HT film from ODCB and then thermally anneal it, we see that the diameter of the crystalline grains increases slightly to $\sim 17 \mathrm{~nm}$ (not shown), a result in agreement with previous reports in the literature. ${ }^{38}$ If we then spin-coat a PCBM overlayer onto the P3HT film, anneal the full bilayer, and then remove the PCBM overlayer by soaking in $\mathrm{CH}$, we recover an almost identical annealed P3HT surface morphology, as shown by the AFM tapping-mode phase image in Figure 1D. This indicates that other than thermal annealing, none of the processing procedures we employ in the fabrication of our solution-processed bilayers affects either the surface morphology or the intrinsic chain packing in the P3HT underlayer and that annealing does not promote intermixing of the two components or alter the intrinsic flatness of the P3HT/PCBM interface.

Now that we know that thermal annealing does not affect the layer structure of our solution-processed bilayers, we can turn to investigate the effects of different processing steps on the morphology of the PCBM overlayer. Figure 4A shows an AFM phase image of the top surface of a $\sim 22 \mathrm{~nm}$ thick PCBM overlayer that was spin-cast from DCM onto a P3HT underlayer. The image is almost perfectly homogeneous, indicating that the film is very flat; the rms surface roughness is only $0.46 \mathrm{~nm}$. The lack of discernible features also shows that the PCBM film is largely amorphous: we would expect a partially crystalline or polycrystalline material to show phase contrast across crystalline domain boundaries because of the difference in force modulus at the edges of the domains, as observed for the P3HT underlayer. In contrast, Figure 4B shows that the surface topography of the PCBM overlayer changes upon thermal annealing, with discernible nanoscale crystallites appearing in the annealed bilayer. The PCBM nanocrystallites are needlelike, with an average length of $43.11 \pm 18.12 \mathrm{~nm}$ and an average width of $8.72 \pm 1.63 \mathrm{~nm}$, and sit in a background of largely amorphous material. Thus, we can conclude that spin-cast PCBM layers are highly amorphous and that thermal annealing induces partial crystallinity in pure PCBM films.

In order to confirm that the topographic features shown in Figure 4B are truly PCBM nanocrystallites, in Figure 4C, we show the results of XRD measurements of both as-cast and thermally annealed spin-coated PCBM films. The blue dotted curve shows that only a weak diffraction peak is observable, centered at $13.8 \mathrm{~nm}^{-1}$, for the as-cast PCBM film, confirming that the as-cast film is primarily amorphous in nature. Upon thermal annealing, the red solid curve shows three distinct peaks at $12.5,13.9$, and $14.7 \mathrm{~nm}^{-1}$. On the basis of the widths of these peaks, the Scherrer equation ${ }^{39}$ gives an estimate for the average diameter of the crystallites of $\sim 20 \mathrm{~nm}$, which is in excellent agreement with the average size of the nanoscale features seen via AFM. The exact assignment of the thermally annealed PCBM diffraction peaks is somewhat difficult to make because there are a number of peaks near these positions in the 


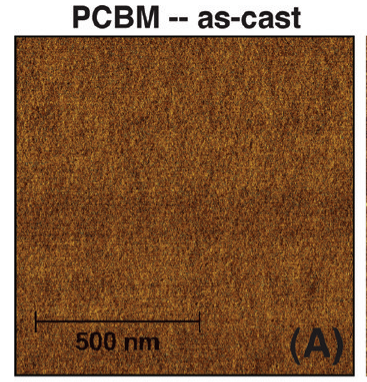

\section{PCBM -- annealed}

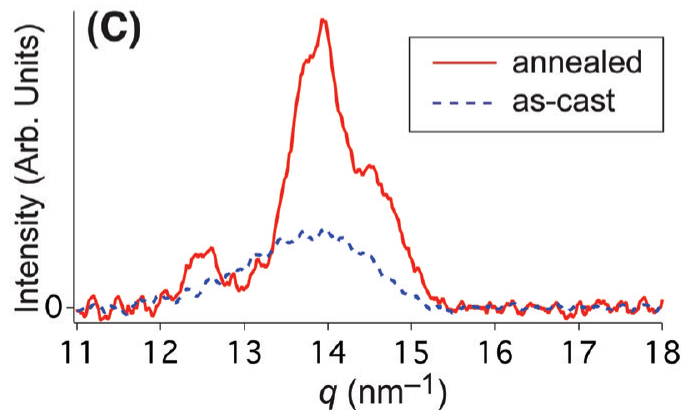

Figure 4. (A) AFM tapping-mode phase image $(1 \mu \mathrm{m} \times 1 \mu \mathrm{m})$ of an as-cast PCBM overlayer spin-cast from DCM on top of a P3HT film. The lack of phase contrast and the $0.46 \mathrm{~nm}$ rms surface roughness indicate that the film is largely amorphous. (B) AFM tapping-mode phase image $(1 \mu \mathrm{m} \times 1 \mu \mathrm{m})$ of a PCBM overlayer spin-cast from DCM on top of a P3HT film after the bilayer had been thermally annealed at $150{ }^{\circ} \mathrm{C}$ for $20 \mathrm{~min}$. Annealing produces PCBM nanocrystallites with an average diameter of $\sim 26 \mathrm{~nm}$ and increases the rms surface roughness of the film to $2.34 \mathrm{~nm}$. (C) XRD of PCBM films spin-cast from DCM both before (blue dashed curve) and after (red solid curve) thermal annealing. The appearance of the diffraction peaks after annealing is consistent with the observation of PCBM nanocrystallites in panel B; the width of these peaks corresponds to an average crystalline domain size of $\sim 20 \mathrm{~nm}$, also in excellent agreement with the AFM results in panel B.

various PCBM crystal structures obtained from the family of polymorphs reported for PCBM crystals grown from different solvents. ${ }^{40}$ Whatever the precise assignment, the data in Figure 4 confirm that spin-cast films of PCBM are quite amorphous and that thermal annealing increases the degree of PCBM crystallinity.

B. Photophysics of Solution-Processed P3HT/PCBM Bilayers. With an understanding of the role thermal annealing plays in the structure of our well-defined bilayers, we turn in Figure 5 to examine the photophysics of our bilayer films. The black squares in Figure 5 show the steady-state PL spectrum of an as-cast $80 \mathrm{~nm}$ thick P3HT film spun from ODCB. The relatively pronounced PL shoulder near $720 \mathrm{~nm}$, along with the highly structured absorption spectrum and red absorption peak near $600 \mathrm{~nm}$ seen in Figure 2, have been attributed by others as resulting from a high degree of order of the $\mathrm{P} 3 \mathrm{HT}$ chains in the film. ${ }^{41}$ This is consistent with our choice of ODCB as the solvent used for spin-coating: ${ }^{42,43}$ the slow evaporation kinetics of ODCB gives the polymer chains more time to aggregate, in accord with the AFM images shown in Figure 1 that verify that the P3HT is highly nanocrystalline. The production of such P3HT aggregrated lamellar phases by slow solvent evaporation or thermal annealing is advantageous for solar-cell operation because more ordered P3HT chains are associated with higher hole mobilities. ${ }^{24,44}$ We note that the absorption spectrum of P3HT films spun from ODCB changes little upon thermal annealing at $150{ }^{\circ} \mathrm{C}$ for $20 \mathrm{~min}$ (not shown), consistent with literature reports: ${ }^{45}$ the slow solvent evaporation of OCDB leaves P3HT films spun from this solvent in an essentially annealed

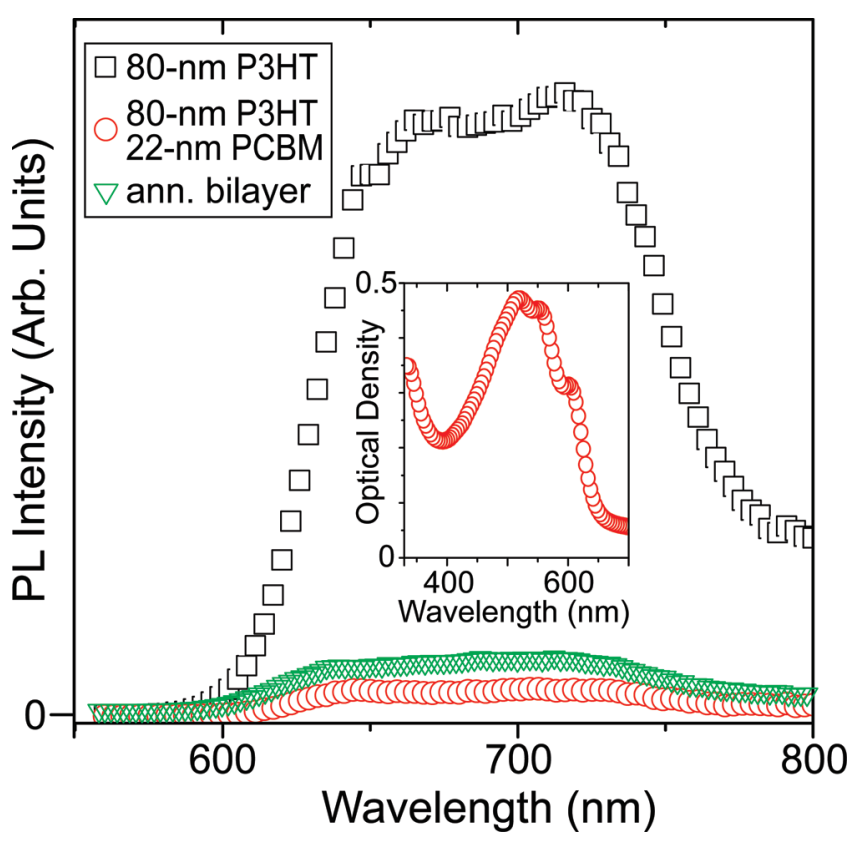

Figure 5. Steady-state PL spectra following $530 \mathrm{~nm}$ excitation for an $80 \mathrm{~nm}$ thick P3HT film spin-cast from ODCB that had had a drop of pure DCM solvent spun on top of it (black squares), a solutionprocessed bilayer with an identical P3HT underlayer and a $22 \mathrm{~nm}$ PCBM overlayer spin-cast from DCM (red circles), and the same bilayer following thermal annealing (green triangles). The spectrally integrated PL quenching of the as-cast P3HT/DCM film upon addition of the PCBM overlayer is $\sim 90 \%$. The inset shows the absorption spectrum of the bilayer prior to thermal annealing.

state. We also note that the residual PL from P3HT:PCBM BHJ blend films spun from ODCB does not display the structure indicative of this high degree of organization because the large amounts of PCBM in such films inhibit ordering of the P3HT chains ${ }^{46}$ consistent with the AFM image shown in Figure 3A. And as discussed above, we saw significant changes in neither the shape nor the intensity of the absorption spectrum of the P3HT layer following deposition of the PCBM overlayer (Figure 5 , inset), either before or after thermal annealing.

The red circles in Figure 5 display the spectroscopy of an as-cast bilayer with an $80 \mathrm{~nm}$ thick P3HT underlayer and a $\sim 22$ $\mathrm{nm}$ thick PCBM overlayer collected under the same conditions as for the pure P3HT film; the layer thicknesses were verified by profilometry. The absorption spectrum of this bilayer, shown in the inset, fits perfectly to the sum of the individual P3HT and PCBM absorption spectra. The PL data in the main panel show clearly that deposition of the thin PCBM overlayer results in highly quenched polymer fluorescence: comparison of the spectrally integrated PL from the bilayer and from the P3HT film with no PCBM overlayer yields a quenching ratio of $\sim 90 \%$. We note that steady-state PL quenching measurements are frequently plagued by thin-film interference effects and/or waveguiding of the fluorescent light. ${ }^{47}$ Although we cannot fully eliminate these effects, we believe they have been minimized by choosing a $\sim 20 \mathrm{~nm}$ thickness for the PCBM overlayer in these experiments, which is so small compared to the wavelength of the emitted light that the presence of the overlayer should not alter any interference or waveguiding effects in the P3HT underlayer. The $\sim 90 \%$ quenching ratio implies a very long effective P3HT exciton quenching length: because the 80 $\mathrm{nm}$ thick P3HT underlayer is photoexcited from the bottom, this result implies an effective quenching length of $\sim 80 \mathrm{~nm}$. This is surprising given that the exciton diffusion length has been estimated by several groups to be in the range of $8-20$ 
TABLE 1: Performance Parameters of ITO/PEDOT:PSS/P3HT/PCBM/Ca/Al Bilayer Solar Cells Under AM-1.5 Illumination

\begin{tabular}{|c|c|c|c|c|c|c|}
\hline $\begin{array}{l}\text { Processing of Solar } \\
\text { Cell Active Bilayer }\end{array}$ & $\mathrm{P}^{3} \mathrm{HT}^{a}(\mathrm{~nm})$ & $\mathrm{PCBM}^{a}(\mathrm{~nm})$ & $J_{\mathrm{sc}}\left(\mathrm{mA} \mathrm{cm}^{-2}\right)$ & $V_{\mathrm{oc}}(\mathrm{V})$ & $\mathrm{FF}(\%)$ & $\mathrm{PCE}^{b}(\%)$ \\
\hline annealed & 50 & 22 & 6.9 & 0.66 & 68 & 3.1 \\
\hline as-cast & 80 & 22 & 6.1 & 0.58 & 39 & 1.4 \\
\hline annealed P3HT only ${ }^{c}$ & 80 & 22 & 5.4 & 0.38 & 38 & 0.8 \\
\hline annealed & 80 & 22 & 7.5 & 0.66 & 68 & 3.4 \\
\hline annealed & 115 & 22 & 6.2 & 0.66 & 53 & 2.2 \\
\hline annealed & 125 & 22 & 6.9 & 0.67 & 54 & 2.5 \\
\hline annealed & 140 & 22 & 6.0 & 0.66 & 45 & 1.8 \\
\hline annealed & 155 & 22 & 4.6 & 0.66 & 40 & 1.2 \\
\hline annealed & 50 & 34 & 3.3 & 0.63 & 52 & 1.1 \\
\hline annealed & 80 & 34 & 5.9 & 0.63 & 68 & 2.5 \\
\hline annealed & 115 & 34 & 8.2 & 0.63 & 66 & 3.5 \\
\hline annealed & 125 & 34 & 8.3 & 0.64 & 64 & 3.4 \\
\hline annealed & 140 & 34 & 8.6 & 0.64 & 63 & 3.5 \\
\hline annealed & 155 & 34 & 8.7 & 0.65 & 61 & 3.4 \\
\hline
\end{tabular}

${ }^{a}$ Thickness of the spin-cast layer. ${ }^{b}$ Power conversion efficiency. ${ }^{c}$ P3HT layer was annealed before deposition of the PCBM overlayer.

nm. ${ }^{6,748}$ We will show in an upcoming paper ${ }^{49}$ that this unusually high PL quenching of thick P3HT films by thin PCBM overlayers results from a novel long-range exciton quenching mechanism. ${ }^{50,51}$

The green triangles in Figure 5 show that upon thermal annealing, the PL intensity from P3HT/PCBM bilayers undergoes a slight increase, indicating a decrease in exciton quenching efficiency. This result is in direct contrast to the work of Drees et al., who observed that thermal annealing led to increased quenching of the PL from interdiffused blends of a poly(phenylene vinylene) derivative and $\mathrm{C}_{60} \cdot{ }^{52}$ These authors attributed this increased quenching as resulting from increased mixing of the polymer and fullerene components induced by thermal annealing. The fact that thermally annealing our bilayer samples results in decreased exciton quenching suggests that annealing does not promote intermixing of the two components in our solution-processed bilayers. ${ }^{19}$ Thus, the behavior of the PL seen in Figure 5, in combination with the data in the previous section, provides consistent evidence that our solution-processed bilayers have a relatively sharp polymer-fullerene interface whether or not they are thermally annealed.

C. Performance Characteristics of Solution-Processed P3HT/PCBM Bilayer Photovoltaics. With an understanding of the morphological and photophysical properties of our solution-processed bilyers in hand, we turn next to the behavior of photovoltaic devices in which these bilayers serve as the active medium. We fabricated bilayer devices with differing P3HT and PCBM layer thicknesses and different annealing conditions, the performance characteristics of which are summarized in Table 1. The solar-cell performance characteristics presented in this table represent average values obtained by testing multiple films with three devices per film. We find an approximately $5 \%$ error in $J_{\mathrm{sc}}$ and $V_{\mathrm{oc}}$ and roughly a $10 \%$ error in FF. Only devices that showed good diodic behavior in the dark were included in the averaging. The injected-current characteristics of some of our bilayer devices are shown in the Supporting Information; if we assume that the current injected is space-charge limited, we extract an average carrier mobility for our devices of $2.3 \times 10^{-5} \mathrm{~cm}^{2} \mathrm{~V}^{-1} \mathrm{~s}^{-1} .36$

1. Effects of Thermal Annealing on Bilayer Solar Cells. Figure 6 shows the performance characteristics of ITO/PEDOT: $\mathrm{PSS} / 80 \mathrm{~nm}$ P3HT/22 nm PCBM/Ca sandwich-structure bilayer solar cells where the active bilayer was either as-cast (black upward triangles) or thermally annealed before deposition of the $\mathrm{Ca}$ cathode (filled blue downward triangles). The data in this figure and Table 1 make it clear that thermally annealing

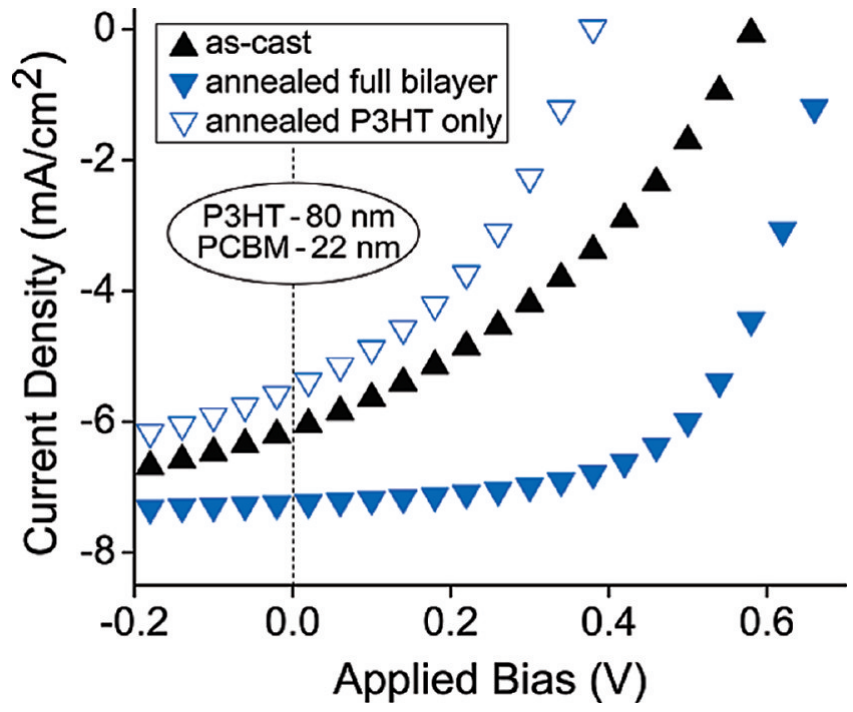

Figure 6. Current density versus applied bias for ITO/PEDOT:PSS/ $\mathrm{P} 3 \mathrm{HT} / \mathrm{PCBM} / \mathrm{Ca} / \mathrm{Al}$ solution-processed bilayer solar cells under $\mathrm{AM}-$ 1.5 illumination, where the active bilayer is either an as-cast $\sim 80 \mathrm{~nm}$ thick P3HT film spin-cast from ODCB with a $22 \mathrm{~nm}$ thick PCBM overlayer spun from DCM (solid black upward triangles) or an $\sim 80$ $\mathrm{nm}$ thick P3HT film with a DCM-spun $\sim 22 \mathrm{~nm}$ PCBM overlayer that had been thermally annealed at $150^{\circ} \mathrm{C}$ for 20 min prior to deposition of the cathode (solid blue downward triangles). For comparison, the open blue downward triangles show the $J-V$ characteristics of an identical bilayer device where the P3HT layer was annealed prior to deposition of both the PCBM overlayer and the cathode. Details of the device performance parameters are summarized in Table 1 .

completed P3HT/PCBM bilayer solar cells prior to Ca deposition dramatically improves the device performance relative to as-cast devices: annealing causes an increase in $J_{\text {sc }}$ of $\sim 23 \%$ and in FF of $\sim 74 \%$ relative to the as-cast device. In addition, annealing produces an increase in the $V_{\text {oc }}$ of these bilayer cells by nearly $15 \%$, which is surprising given that annealing has been shown to have relatively little effect on the $V_{\text {oc }}$ of BHJ cells fabricated from these same materials. ${ }^{20,53} \mathrm{We}$ are confident that these annealing-induced changes in the performance of the bilayer cells do not result from changes in the morphology of the P3HT layer for two reasons. First, we saw very little change in the P3HT absorption spectrum or surface topography upon annealing, suggesting that thermal annealing does little to change the degree of chain ordering in the highly organized P3HT layer that was cast from ODCB. ${ }^{45}$ Second, we also prepared bilayer solar cells in which we annealed the P3HT underlayer before 
spin-coating the PCBM overlayer, and we found that the performance of these devices decreased relative to those in which neither layer was annealed, as shown by the open blue downward triangles in Figure 6. Thus, the thermal annealinginduced improvement in performance of our bilayer devices is most likely due to changes that occur within the fullerene layer.

We believe that the performance improvements that occur in bilayer devices that were thermally annealed result primarily from the annealing-induced increase in crystallinity of the fullerene overlayer (cf. Figure 4). This is because the morphology of the as-cast fullerene layer is largely amorphous, a consequence of kinetic trapping of the interfullerene packing structure due to the rapid evaporation of DCM during spincoating. The amorphous nature of this layer results in a large degree of positional and energetic disorder, which is correlated with slow electron-hopping rates and thus poor electron mobility. The partial crystallization of PCBM that occurs upon annealing removes some of this disorder, producing an increased electron mobility and hence an increased photocurrent. The increased electron mobility also decreases the average transit time $\left(t_{\mathrm{tr}}\right)$ for the electrons to traverse the fullerene layer. ${ }^{54}$ As discussed further below, we believe that the annealing-induced improvement in fill-factor is a direct consequence of an improved balance of the carrier transit times for the electrons and holes in the bilayer device.

2. Effects of Layer Thickness on the Performance of Bilayer Solar Cells. The data in the previous section argue strongly that electron mobility in the PCBM layer plays a significant role in determining the shape of the current-voltage curve of solution-processed P3HT/PCBM bilayer solar cells. To better understand the roles of electron mobility and perhaps most importantly the balance of carrier transit times on device performance, we have measured the operating characteristics of bilayer solar cells as a function of the thickness of the individual P3HT and PCBM layers, as summarized in Figure 7 and Table 1. Because all of the carriers in bilayer photovoltaics are generated within a few nanometers of the donor-acceptor interface, our ability to vary the thickness of the individual layers allows us to study how changing the transit time of each carrier affects solar cell performance without significantly changing the bulk mobility of either component or the nature of the interfaces. This is something that is not possible with BHJ devices, where changes in the nanometer-scale morphology of the interpenetrating network of the two components with processing conditions ${ }^{1,4}$ make it impossible to determine the distance carriers must traverse to exit the device or to keep the mobility of one carrier virtually fixed while varying the mobility of the other carrier via thermal annealing.

Figure 7A shows the AM-1.5-illuminated current-voltage characteristics of P3HT/PCBM bilayer solar cells annealed before deposition of the $\mathrm{Ca}$ cathode with three different $\mathrm{P} 3 \mathrm{HT}$ layer thicknesses: $50 \mathrm{~nm}$ (green diamonds), $80 \mathrm{~nm}$ (blue triangles), and $115 \mathrm{~nm}$ (red squares). The PCBM layer thickness was held fixed at $22 \mathrm{~nm}$ for all of these devices. The data show that the photovoltaic power conversion efficiency does not change monotonically with P3HT layer thickness: the efficiency of the devices with $80 \mathrm{~nm}$ thick P3HT layers is higher than those of the devices with thicker and thinner P3HT layers. We believe that this P3HT thickness dependence of the device performance results from a trade-off between improved optical absorption and misbalanced carrier transit times ${ }^{54}$ as the thickness of the P3HT layer is increased. The devices with 50 $\mathrm{nm}$ thick P3HT layers have the highest fill-factor, suggesting that the carrier transit times in these cells are the closest to being
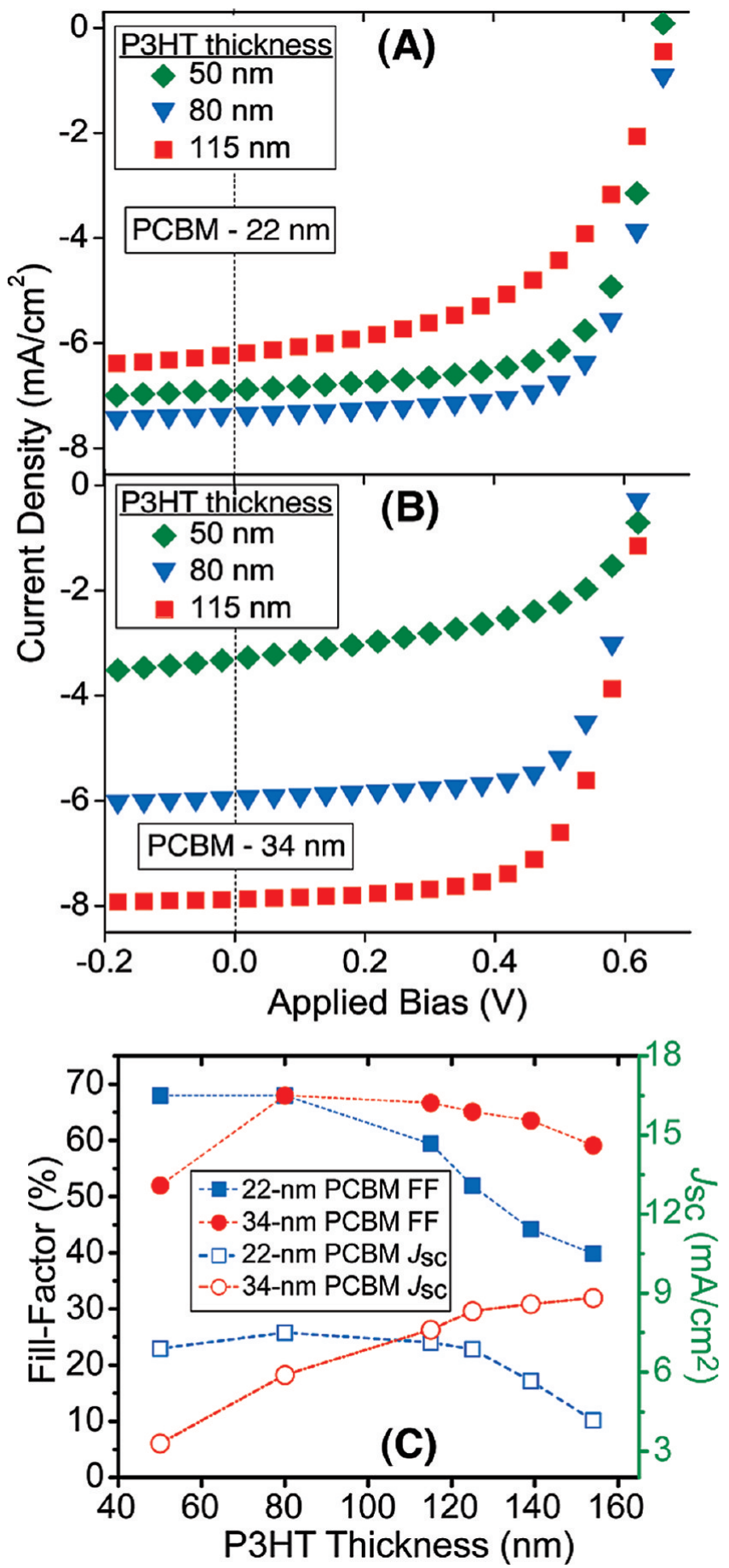

Figure 7. (A) Current density versus applied bias for thermally annealed ITO/PEDOT:PSS/P3HT/PCBM/Ca/Al solution-processed bilayer solar cells under AM-1.5 illumination with a $22 \mathrm{~nm}$ thick PCBM overlayer for different thicknesses of the P3HT underlayer spun from ODCB: $50 \mathrm{~nm}$ (green diamonds), $80 \mathrm{~nm}$ (blue triangles), and $115 \mathrm{~nm}$ (red squares). (B) The same as panel A but for devices with a $34 \mathrm{~nm}$ thick PCBM overlayer spun from DCM. (C) Bilayer-solar-cell performance factors as a function of P3HT layer thickness. The squares show the performance of devices with a $22 \mathrm{~nm}$ thick PCBM layer, whereas the circles show the performance of devices with a $34 \mathrm{~nm}$ thick PCBM layer. Solid symbols denote the device fill-factor (left axis), whereas open symbols denote the device short-circuit current (right axis). Lines connecting the data points are meant to guide the eye. Details of the device performance parameters are summarized in Table 1.

optimally balanced. The overall efficiency of these devices is thus likely limited only by photon harvesting, because the thin $50 \mathrm{~nm}$ polymer layer has an optical density at the P3HT 
absorption maximum of only $\sim 0.35$. When the thickness of the P3HT layer is increased to $80 \mathrm{~nm}$, the $J_{\text {sc }}$ increases because the larger absorption by the thicker polymer layer leads to the creation of additional carriers. The fill-factor of the $80 \mathrm{~nm}$ thick $\mathrm{P} 3 \mathrm{HT}$ devices is slightly lower than those of the $50 \mathrm{~nm}$ devices, however, suggesting that the increased hole transit time associated with the thicker P3HT layer is becoming out of balance with the smaller electron transit time in the thin PCBM layer. When the device thickness is further increased to $115 \mathrm{~nm}$, even though the absorption is further increased, the hole transit time becomes so out of balance with the electron transit time that the FF decreases considerably, and the $J_{\mathrm{sc}}$ is significantly reduced. Another possible explanation for the poor performance of this device is that the $115 \mathrm{~nm}$ thick polymer layer has become much larger than the effective quenching length, limiting exciton harvesting and thus device efficiency. The PL quenching data in Figure 5, however, suggest that exciton harvesting is not a problem in our bilayer samples. ${ }^{51}$ We will show next that it is indeed misbalanced carrier transit times that limit the performance of bilayer devices with thick P3HT layers.

If misbalanced carrier transit times are really the main culprit limiting the fill-factor in P3HT/PCBM bilayer solar cells with thick P3HT layers, then it should be possible to improve devices with thick P3HT layers by increasing the thickness of the PCBM layer to improve the balance. Figure 7B shows the photovoltaic performance of a set of P3HT/PCBM bilayer solar cells fabricated under conditions identical to those in Figure 7A except that the PCBM overlayer thickness was increased from 22 to $34 \mathrm{~nm}$. The simple act of increasing the PCBM layer thickness leads to a completely different trend of the power conversion efficiency with P3HT layer thickness: with the 34 $\mathrm{nm}$ thick PCBM overlayer, it is the devices made with $115 \mathrm{~nm}$ thick P3HT layers (solid red squares) that have by far the best power conversion efficiency, despite the fact that one might expect there to be poor diffusion of the $\mathrm{P} 3 \mathrm{HT}$ excitons to the PCBM interface through such a thick layer of P3HT. ${ }^{6}$ In fact, these devices have a higher $J_{\mathrm{sc}}$ than any of the devices with the $22 \mathrm{~nm}$ thick PCBM layer shown in Figure 7A, including the devices with $80 \mathrm{~nm}$ thick P3HT layers (solid blue triangles), and have power conversion efficiencies that exceed 3.5\%. The fact that the $115 \mathrm{~nm}$ P3HT/34 nm PCBM bilayer devices have a higher current, a similar FF, and a higher efficiency than comparable devices in which both layers are thinner indicates that it is balancing the carrier transient times-not simply minimizing them-that is important for the optimization of polymer-fullerene solar cells. The devices in Figure 7B with thinner P3HT layers thus suffer from both a reduced absorption and a more misbalanced set of carrier transit times, explaining their lower net power conversion efficiency. ${ }^{55} \mathrm{We}$ show in the Supporting Information that the effective mobility of the carriers injected into bilayers in the dark is also improved when the carrier transit times are balanced, even when the total device thickness has to be increased to achieve this balance. ${ }^{36}$

Figure 7C and Table 1 summarize how both the fill-factor (filled symbols) and the short-circuit current (open symbols) of our bilayer devices vary with P3HT thickness for bilayers with both $22 \mathrm{~nm}$ (blue squares) and $34 \mathrm{~nm}$ (red circles) PCBM overlayers. Because the open-circuit voltage is nearly the same for all of these devices, the device efficiencies are proportional to the product of the short-circuit current and the fill-factor (Table 1). This figure shows clearly how the fill-factor reaches a maximum when the P3HT thickness is chosen to match the carrier transit times in the two layers, as discussed above. But perhaps the most striking feature of the data in Figure $7 \mathrm{C}$ is that for the devices with the $34 \mathrm{~nm}$ thick PCBM overlayer, the short-circuit current continues to increase with P3HT layer thickness, even for P3HT layers as thick as $150 \mathrm{~nm}$. This again indicates that exciton harvesting is not what limits the performance of our bilayer devices and suggests that we could make even more efficient bilayer devices if we were able to spincoat PCBM layers that were thicker than $34 \mathrm{~nm}$.

The other important feature of the data in Figure 7 is that for a given PCBM overlayer thickness, the optimally efficient bilayer solar cell is the one with a P3HT layer that is about four times thicker than the PCBM layer. Because the distance the holes must travel to be extracted is four times that of the electrons, this strongly indicates that the mobility of the electrons in the PCBM layer is smaller than that of the holes in the P3HT layer: in other words, it is the conduction and/or extraction of electrons that ultimately limits the performance of these devices. We believe that the mobility of electrons in the PCBM layer is the limiting factor because bilayer devices with annealed PCBM layers work better than bilayer devices with as-cast PCBM layers, independently of the state of the P3HT layer (Figure 6, Table 1). We also have argued in previous work that it is electron conduction among the fullerene component of P3HT: PCBM BHJ devices that is performance-limiting; ${ }^{19}$ those arguments are reinforced by the data given here. In other words, the BHJ geometry forces electrons to travel a longer, more tortuous path on a PCBM network that is much less crystalline than is the case in our annealed bilayer devices. Because the BHJ geometry involves an interpenetrating network, the fullerene thickness cannot be adjusted independently of the polymer thickness as it can in the bilayer geometry. Thus, these results imply that the most fruitful avenues for investigating how to further improve polymer-fullerene solar cells lie in improving carrier mobility in the fullerene component, not the polymer component, of the devices. ${ }^{56}$

\section{Conclusions}

In summary, we have prepared fully solution-cast P3HT/ PCBM bilayer solar cells with well-defined planar interfaces and found that their photovoltaic performance rivals that of $\mathrm{BHJ}$ devices fabricated from the same materials. We found that ODCB and DCM serve as an excellent pair of orthogonal solvents for the sequential spin-coating of regioregular P3HT and PCBM layers, respectively, and that spinning the PCBM overlayer has essentially no effect on the morphology of the P3HT underlayer. The ease of solution processing not only provides a general method for fabricating bilayer devices from materials that do not survive thermal deposition but also allows the performance of each layer to be optimized (e.g., via thermal annealing or use of solvent additives) individually, something that is not possible for devices based on the BHJ architecture.

Our choice to fabricate bilayer $\mathrm{P} 3 \mathrm{HT} / \mathrm{PCBM}$ devices is based on the fact that their geometry is much simpler than the complex nanometer-scale architecture inherent in BHJ devices. In particular, the nature of the interpenetrating network in BHJ devices is quite sensitive to the degree of mixing of the two components in the blend film, which in turn depends critically on the processing conditions. This has made it challenging to fully understand the changes in photovoltaic performance observed upon the thermal annealing of BHJ devices because annealing simultaneously changes the mobilities of both carriers, likely in opposite directions. ${ }^{19}$ Annealing also changes the effective carrier pathlengths and transit times, as well as the nature of any extraction barriers at the organic/electrode interfaces. By studying bilayers with a controllably fixed 
geometry, we have been able to isolate the effect of misbalancing the carrier transit times on device performance.

One of the advantages of the bilayer geometry is that the stepwise deposition of the bottom and top layers allows the distance that each of the carriers traverses to be controlled independently, without simultaneously changing either the carrier mobility or the nature of any of the interfaces. We found that the highest fill-factors in bilayer devices are achieved not by minimizing the electron and hole transit times but by balancing them. The idea of increasing the thickness of the active layer to improve charge transport is counterintuitive for BHJs: the thicker the BHJ, the longer it takes carriers to escape the active layer, and thus, the more likely it is to lose significant numbers of carriers to bimolecular recombination. ${ }^{29}$ For bilayers, however, bimolecular recombination is not a significant issue, and thus, the thickness of the active layers can be greatly increased as long as the balance in carrier transit times is maintained. When the carrier transit times are balanced, we can produce devices with fill-factors of $70 \%$ and AM-1.5 power conversion efficiencies in excess of $3.5 \%$.

We close by highlighting that the bilayer devices with the highest efficiencies contain a P3HT underlayer that is roughly four times thicker than that of the PCBM overlayer, which implies that it is the conduction and extraction of electrons through the fullerene layer that limit the performance of both bilayer and BHJ devices based on these materials: because the path that electrons must traverse in BHJ films is much more tortuous and less crystalline than that in the thin fullerene overlayer in a bilayer, it makes sense that electron transport on the PCBM component is what limits the performance of BHJ solar cells. ${ }^{19}$ This argument is also supported by the fact that improved crystallinity of the PCBM overlayer is responsible for the improvements in bilayer device performance upon thermal annealing. Thus, we believe that the greatest potential for improving the performance of polymer-based photovoltaics lies in using electron acceptors with higher charge-carrier mobilities and finding a suitable way to optimize electron extraction at the cathode. We also note that the most efficient bilayer devices described here had P3HT layers that were $\sim 115$ $\mathrm{nm}$ thick, an order of magnitude larger than the canonical value assumed for the exciton diffusion length in P3HT. ${ }^{6}$ Given that exciton harvesting in bilayers appears to be more efficient than previously thought, ${ }^{49}$ we also believe that the bilayer geometry offers a better means to accomplish these goals than the kinetically trapped nanoscale complexity inherent in BHJs.

Acknowledgment. We would like to thank Ian M. Craig for help with profilometry measurements. This work was supported by the Office of Naval Research under Contract no. N0001404-1-0410 and the National Science Foundation under Grant no. CHE-0527015.

Supporting Information Available: This information includes electron-microscopy images on cross sections of our bilayers, injected-current characteristics of some of our bilayer devices, and effective mobilities of carriers injected into bilayers in the dark. This material is available free of charge via the Internet at http://pubs.acs.org.

\section{References and Notes}

(1) Dennler, G.; Scharber, M.; Brabec, C. J. Adv. Mater. 2009, 21 , 1323.

(2) Yao, Y.; Hou, J.; Xu, Z.; Li, G.; Yang, Y. Adv. Funct. Mater. 2008, $18,1783$.

(3) Hwang, I.; Cho, S.; Kim, J.; Lee, K.; Coates, N.; Moses, D.; Heeger, A. J. J. Appl. Phys. 2008, 104, 033706.
(4) Günes, S.; Neugebauer, H.; Sariciftci, N. S. Chem. Rev. 2007, 107, 1324.

(5) Yang, C. Y.; Heeger, A. J. Synth. Met. 1996, 83, 85.

(6) Shaw, P.; Ruseckas, A.; Samuel, I. Adv. Mater. 2008, 20, 3516.

(7) Huijser, A.; Savenije, T. J.; Shalav, A.; Siebelles, L. D. J. Appl. Phys. 2008, 104, 034505.

(8) Kraabel, B.; Hummelen, J. C.; Vacar, D.; Moses, D.; Sariciftci, N. S.; Heeger, A. J.; Wudl, F. J. Chem. Phys. 1996, 104, 4267.

(9) Muller, J. G.; Scharber, M.; Lemmer, U.; Feldmann, J.; Scherf, U.; Sariciftci, N. S. Synth. Met. 2003, 137, 1475.

(10) Nogueira, A. F.; Montanari, I.; Nelson, J.; Durrant, J. R.; Winder, C.; Sariciftci, N. S. J. Phys. Chem. B 2003, 107, 1567.

(11) Cook, S.; Katoh, R.; Furube, A. J. Phys. Chem. C 2009, 113, 2547.

(12) Yoshino, K.; Tada, K.; Fujii, A.; Hosoda, K.; Kawabe, S.; Kajii, H.; Hirohata, M.; Hidayat, R.; Araki, H.; Zakhidov, A. A.; Sugimoto, R.; Iyoda, M.; Ishikawa, M.; Masuda, T. Fullerene Sci. Technol. 1997, 5, 1359.

(13) Bakulin, A. A.; Martyanov, D. S.; Paraschuk, D. Y.; Pshenichnikov,

M. S.; van Loosdrecht, P. H. M. J. Phys. Chem. B 2008, 112, 13730.

(14) Gregg, B. A. J. Phys. Chem. B 2003, 107, 4688.

(15) Ma, W.; Yang, C.; Gong, X.; Lee, K.; Heeger, A. J. Adv. Func. Mater. 2005, 15, 1617.

(16) Chen, H. Y.; Yang, H. C.; Yang, G. W.; Sista, S.; Zadoyan, R. B.; Li, G.; Yang, Y. J. Phys. Chem. C 2009, 113, 7946.

(17) Reyes-Reyes, M.; Kim, K.; Carroll, D. Appl. Phys. Lett. 2005, 87, 083506.

(18) Park, S. H.; Roy, A.; Beauptre, S.; Cho, S.; Coates, N.; Moon, J. S.; Moses, D.; Leclerc, M.; Lee, K.; Heeger, A. J. Nat. Photonics 2009, 3, 297.

(19) Ayzner, A. L.; Wanger, D. D.; Tassone, C. J.; Tolbert, S. H.; Schwartz, B. J. J. Phys. Chem. C 2008, 112, 18711.

(20) Mihailetchi, V. D.; Xie, H.; de Boer, B.; Koster, L. J. A.; Blom, P. W. M. Adv. Funct. Mater. 2006, 16, 699.

(21) Von Hauff, E.; Parisi, J.; Dyakonov, V. Thin Solid Films 2006, 511,506 .

(22) Dante, M.; Peet, J.; Nguyen, T. Q. J. Phys. Chem. C 2008, 112, 7241

(23) Ballantyne, A. M.; Chen, L.; Dane, J.; Hammant, T.; Braun, F. M.; Heeney, M.; Duffy, W.; McCulloch, I.; Bradley, D. D. C.; Nelson, J. Adv. Funct. Mater. 2008, 18, 2373.

(24) Yang, X.; van Duren, J. K. J.; Rispens, M. T.; Hummelen, J. C.; Janssen, R. A. J.; Michels, M. A. J.; Loos, J. Adv. Mater. 2004, 16, 802.

(25) Savenije, T. J.; Kroeze, J. E.; Yang, X.; Loos, J. Adv. Funct. Mater. $\mathbf{2 0 0 5}, 15,1260$.

(26) Van Bavel, S. S.; Sourty, E.; de With, G.; Loos, J. Nano Lett. 2009, $9,507$.

(27) Coffey, D. C.; Reid, O. G.; Rodovsky, D. B.; Bartholomew, G. P.; Ginger, D. S. Nano Lett. 2007, 7, 738.

(28) Chu, C.; Yang, H.; Hou, W.; Huang, J.; Li, G.; Yang, Y. Appl. Phys. Lett. 2008, 92, 103306.

(29) Groves, C.; Marsh, R. A.; Greenham, N. C. J. Chem. Phys. 2008, 129,114903

(30) Ferenczi, T. A. M.; Nelson, J.; Belton, C.; Ballantyne, A. M.; Campoy-Quiles, M.; Braun, F. M.; Bradley, D. D. C. J. Phys.: Condens. Matter 2008, 20, 475203.

(31) Kim, B. J.; Miyamoto, Y.; Ma, B.; Frechet, J. M. Adv. Mater. 2009, 19,1

(32) O’Brien, D.; Weaver, M. S.; Lidzey, D. G.; Bradley, D. D. C. Appl. Phys. Lett. 1996, 69, 881.

(33) Shrotriya, V.; Li, G.; Yao, Y.; Moriarty, T.; Emery, K.; Yang, Y. Adv. Funct. Mater. 2006, 16, 2016.

(34) Dennler, G.; Prall, H.-J.; Koeppe, R.; Egginger, M.; Autengruber, R.; Sariciftci, N. S. Appl. Phys. Lett. 2006, 89, 073502.

(35) Aryal, M.; Buyukserin, F.; Mielczarek, K.; Zhao, X.-M.; Gao, J.; Zakhidov, A.; Hu, W. J. Vac. Sci. Technol. B 2008, 26, 2562.

(36) Supplementary Information available on the web at http://acs.pubs. org, 10.1021/jp9050897.

(37) Li, G.; Shrotriya, V.; Yao, Y.; Huang, J.; Yang, Y. J. Mater. Chem 2007, 17, 3126.

(38) Yang, X.; Loos, J.; Veensra, S. C.; Verhees, W. J. H.; Wienk, M. M.; Kroon, J. M.; Michels, M. A. J.; Janssen, R. A. J. Nano Lett. 2005, 5,579 .

(39) Cullity, B. D. Elements of X-ray Diffraction 101-102; AddisonWesley: Reading, MA, 1978.

(40) Rispens, M. T.; Meetsma, A.; Rittberger, R.; Brabec, C. J.; Sariciftci, N. S.; Hummelen, J. C. J. Chem. Commun. 2003, 17, 2116.

(41) Jiang, X. M.; Österbacka, R.; An, C. P.; Vardeny, Z. V. Synth. Met. 2003, 137, 1465 .

(42) Zheng, X.; Chen, L.-M.; Yang, G.; Huang, C.-H.; Hou, J.; Wu, Y.; Li, G.; Hsu, C.-S.; Yang, Y. Adv. Funct. Mater. 2009, 19, 1227.

(43) Li, G.; Yao, Y.; Yang, H.; Shrotriya, V.; Yang, G.; Yang, Y. Adv. Funct. Mater. 2007, 17, 1636.

(44) Cho, S.; Lee, K.; Yuen, J.; Wang, G.; Moses, D.; Heeger, A. J.; Surin, M.; Lazzaroni, R. J. Appl. Phys. 2006, 100, 114503. 
(45) Janssen, G.; Aguire, A.; Goovaets, E.; Vanleake, P.; Poortmans, J.; Manca, J. J. Eur. Phys. J. Appl. Phys 2007, 37, 287.

(46) Shrotriya, V.; Ouyang, J.; Tseng, R. J.; Li, G.; Yang, Y. Chem. Phys. Lett. 2005, 411, 138.

(47) Scully, S. R.; McGehee, M. D. J. Appl. Phys. 2006, 100, 033907. (48) Kroeze, J. E.; Savanije, T. J.; Vermeulen, M. J. W.; Warman, J. M. J. Phys. Chem. B 2003, 107.

(49) Ayzner, A. L.; Kilbride, D.; Tremolet de Villers, B.; Tassone, C. J.; Tolbert, S. H., Rubin, Y.; Schwartz, B. J., manuscript in preparation. (50) Lloyd, M. T.; Lim, Y.-F.; Malliaras, G. G. Apply. Phys. Lett. 2008, 92,143308

(51) Collini, E.; Scholes, G. D. J. Phys. Chem. A 2009, 113, 4223.

(52) Drees, M.; Premaratne, K.; Graupner, W.; Heflin, J. R.; Davis, R. M.; Marciu, D.; Miller, M. Apply. Phys. Lett 2002, 81, 4607.

(53) Vanlaeke, P.; Vanhoyland, G.; Aernouts, T.; Cheyns, D.; Deibel, C.; Manca, J.; Heremans, P.; Poortmans, J. Thin Solid Films 2006, 511, 358
(54) We define the $t_{\text {tr }}$ as the time for carriers to be extracted from the device once they are created. For a bilayer device, this is the time it takes carriers to drift from the $\mathrm{P} 3 \mathrm{HT} / \mathrm{PCBM}$ interface where they are created to the electrodes, which should depend directly on the thickness of the organic layer that they traverse.

(55) We note that minimizing the carrier transit times is likely to be much more important in BHJ devices, where the strong degree of mixing of the two components allows recombination to have a significant role the longer carriers take to escape. Recombination is much less of an issue in bilayers, because oppositely charged carriers never re-encounter each other once they leave the P3HT/PCBM interface.

(56) Kennedy, R.; Ayzner, A. L.; Wanger, D. D.; Day, C. T.; Halim, M.; Khan, S. I.; Tolbert, S. H.; Schwartz, B. J.; Rubin, Y. J. Am. Chem. Soc. 2008, 130, 17290.

JP9050897 